\title{
Scar Revision and Secondary Reconstruction for Skin Cancer
}

\author{
Michael J. Brenner ${ }^{1,2}$ and Jennifer L. Nelson ${ }^{2}$ \\ ${ }^{1}$ Director of Facial Plastic \& Reconstructive Surgery \\ 2Division of Otolaryngology, Department of Surgery \\ Southern Illinois University School of Medicine
}

USA

\section{Introduction}

Late wound management requires not only mastery of the techniques involved in scar revision, but a thorough understanding of facial anatomy, wound healing, and the psychological factors associated with traumatic injury. Treatment of a patient for scar revision requires the surgeon to understand that a patient's perception of a scar is often influenced by emotionally charged circumstances and possible self-critical evaluation. This chapter addresses the etiology, evaluation, and treatment of traumatic wounds in the delayed setting with emphasis on scar revision.

\section{Pathogenesis of scar formation}

Unsightly scar formation and impaired wound healing may arise from a variety of factors related to trauma, surgery, or inflammation.(1) While the stigmata of trauma often appear isolated to the skin, many deformities also involve deeper injury to muscle, bone, or other underlying deep tissues. This distinction is of paramount importance because failure to appropriately identify a structural defect in the scaffolding and supporting tissue that is deep to the skin will almost certainly result in an unfruitful attempt at revision. Furthermore certain types of injuries, such as gunshot wounds, avulsions, and full thickness burns are associated with significant tissue loss.

Several additional factors also adversely affect healing. Infection in the wound bed will exacerbate the degree of injury and will likely to cause added delay in revision. Infected wounds are characterized by greater tissue loss and destruction, as well as increased collagen deposition, impaired vascular supply, and worse scarring. Blunt injuries tend to cause more diffuse soft tissue injury than sharp injuries. Crush injuries that produce stellate tears, irregular lacerations, and diffuse underlying soft tissue destruction may result in particularly severe scarring. Host factors, such as skin thickness, predisposition to keloid formation or hypertrophic scars, skin pigmentation, prior injury, poor nutritional status, sun exposure, and smoking history will all also affect healing and scar formation.

The initial management of a traumatic wound heavily impacts the need for revision.(2) Wound closure is often performed by personnel with limited experience in plastic surgical 
technique and wound management. As a result, wounds may be inadequately cleansed, with devitalized tissue and foreign body contamination predisposing to infection and inflammation. Conversely, overzealous debridement may result in an uneven or irregular wound. The wound closure may be inadequate or traumatic, and widened scars occur over sites of excess tension. Depressed scars occur if wound edges are not appropriately everted. When wounds are not covered with an occlusive dressing or appropriate ointment, desiccation will impair wound healing. Last, those, wounds that are situated at sites of repeated motion are prone to widening and delayed repair.

\section{Evaluation of late wounds}

Successful late wound management is predicated upon a thorough history and evaluation of the patient considering both the location and characteristics of the wound as well as the goals and expectations of the patient.(3) Preoperative photography plays an important role in documenting the extent of disfigurement. Patients need to be reminded that while treatments may camouflage pathologic wound healing, most interventions will exchange one type of scar or deformity for another, lesser one.(4) The indications for delayed wound management relate to an unacceptable appearance or a functionally problematic healing outcome.(5) Evaluation is influenced by anatomic site, mechanism of injury, extent of the deformity, and likelihood of pathologic healing.

\subsection{Characteristics of disfiguring scars}

Scars are perceived as unsightly when their surface characteristics differ markedly from the surrounding skin such that they are poorly camouflaged by the surrounding surface skin anatomy. Whereas scars that fall into shadows generally appear hidden, scars that traverse a smooth convexity such as the chin or malar eminence will be readily noticeable. Abnormal color, contour, and texture make scars more conspicuous and unsightly. Scars that are widened, long, and linear will similarly draw attention, particularly when they are unfavorably oriented relative to relaxed skin tension lines or disrupt an aesthetic subunit. Not uncommonly, cosmetically disfiguring scars are also associated with functional problems, such as contracture, distortion, stenosis, or fistula formation. Some examples are ectropion, entropion, or webbing of the eyelids; disruption of salivary ducts; and deformity of the nasal alae, ears, or lips.

\subsubsection{Scar color, contour, and texture}

Poor color match results from hyperpigmentation or hypopigmentation. Hyperpigmented scars have a deep red hue from inflammation or have darkening from increased melanin. Hypopigmentation reflects a loss of melanocytes and tend to be irreversible. Traumatic tattooing occurs when dirt, asphalt, graphite, or other foreign material is embedded within the skin. These particles can be particularly difficult to remove because they tend to be distributed across several different skin layers. Scars that are hypertrophied, elevated, depressed, or that have other poor contour are also difficult to mask, especially when accompanied by webbing or pin cushioned appearance. Unacceptable appearance also results from poor texture, such as a scar that is too shiny or too smooth. 


\subsubsection{Scar length}

Long, linear scars are usually more problematic than shorter scars with more segments because their regular appearance is readily discerned. The human eye has more difficulty detecting a scar's full extent if there is intervening normal tissue or irregularity to the scar. In addition, it is common for long scars to have a bowstring effect over sites such as the medial canthus or mandible to the neck, where webbing can occur due to concavity of these areas. In addition, muscle action can exaggerate a linear deformity, as in the example of curved scars that form a trapdoor deformity.

\subsubsection{Scar depth}

Deeper injuries induce greater scar formation than shallow injuries as a result of the correspondingly greater soft tissue contracture and volume loss. The underlying mechanism involves the melding of superficial and deep scar, resulting in tethering and visible depression. Multiple depths of injury will multiply the extent of scaring, with stellate or crushing injuries resulting in worse injury. Avulsion of tissue will further complicate healing, making it impossible to align skin edges at time of initial injury. Deep, beveled injuries maximize the amount of dermal trauma due to the correspondingly greater area of tissue traversed and the tendency of oblique contracture of the dermis to cause one skin edge to slide over the other. This pattern of scarring may cause either a pin cushioned appearance or a heaped appearance. In such cases, the surgeon may either debulk the elevated skin and place bolster sutures or fully excise the affected area.

\subsubsection{Relation to relaxed skin tension lines (RSTLs)}

Relaxed skin tension lines (RSTLs) run perpendicular to the direction of maximal underlying tension within the skin (Figure 1). Those scars that are unfavorably positioned relative to RSTLs are most likely to require revision. Often the approach to scar revision is based upon how the scar can be reoriented to fall within these lines. The ability to align scars in this manner is often the difference between an excellent and mediocre result because placement within RSTLs improves camouflage and enables the contractile forces on the skin to approximate wound edges, rather than distracting the edges apart. The lines of maximum extensibility (LMEs) run perpendicular to the RSTLs and are usually parallel to muscle fibers. Lines of maximum extensibility are important to consider when recruiting tissue from adjacent areas for flap reconstruction.

Relaxed skin tension lines generally lie perpendicular to the underlying muscle fibers; but, this rule is not absolute. The RSTLs reflect tension on the skin that arises not only from muscle forces but also from stretch by soft tissue or rigid bone/cartilage. Similarly, wrinkle lines do not always accurately reflect the positions of the RSTLs. For example, the lines between the lower lip and mentum run parallel to the orbicularis oris muscle. Most of the RSTLs are parallel to 4 main facial lines: the facial median, the nasolabial, the facial marginal, and the palpebral lines. The facial median line spans from the alar facial groove to the columella and lip and then inferiorly to the mentum. The nasolabial line runs from the alar facial groove inferolaterally to form the nasolabial fold, traverses lateral to the oral commissure, and then extends inferiorly to form marionette lines. The facial marginal line 
starts at the hairline, travels anterior to the tragus, and descends along the posterior margin of the mandible, across the submandibular triangle, and to the hyoid. The palpebral line extends from the superolateral dorsum to the medial canthus and then proceeds to the lateral canthus to the cheek and submental area.

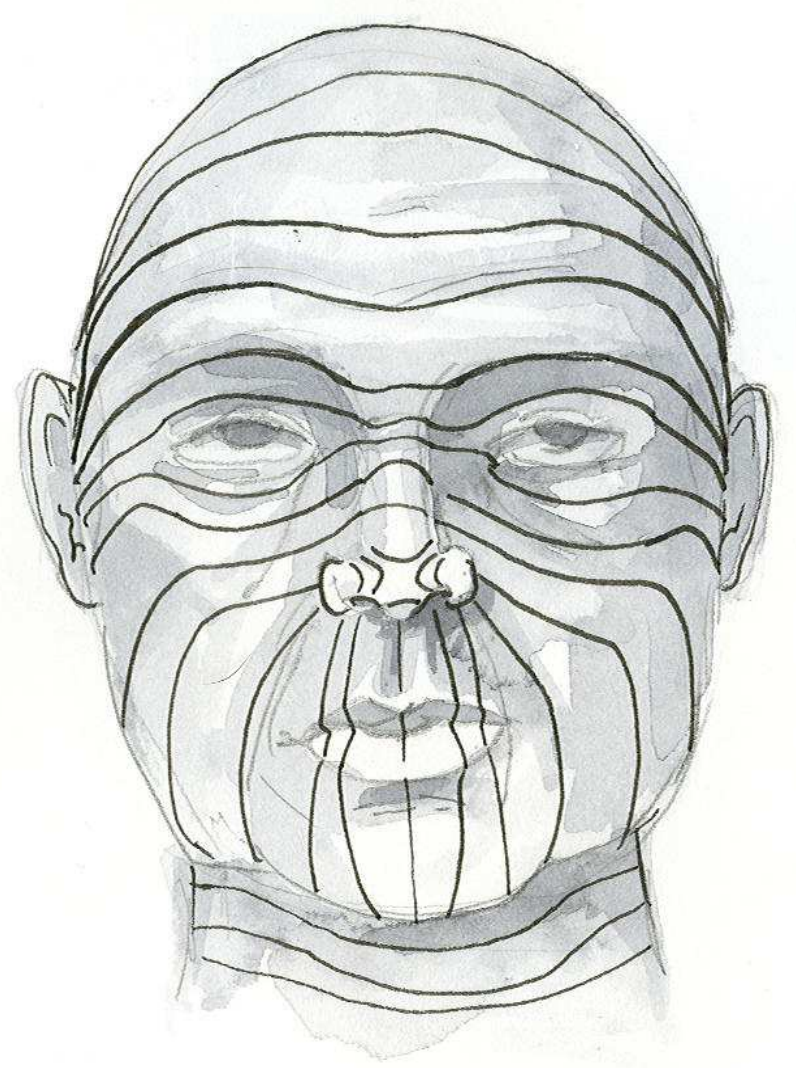

Fig. 1. Relaxed Skin Tension Lines.

\subsection{Relative contraindications to scar revision}

While many patients will benefit from surgical scar revision, several considerations must be taken into account including medical co-morbidities and the prospects for achieving a favorable visible outcome. It is preferable to avoid operating on immature scar, and the surgeon must use judgment when a patient presses for an inappropriately timed surgical intervention. Consideration must also be given to the psychological preparedness of the patient with attention to any unrealistic expectations that the patient may not have disclosed initially. Cigarette smoking should be discontinued at least 2 weeks prior to surgery. Use of nonsteroidal anti-inflammatory agents, Vitamin E, and herbal preparations that may impair wound healing should also be discontinued perioperatively. 


\subsection{Timing and psychological considerations}

The time course of scar maturation is approximately 12 to 18 months, during which a complex sequence of histological changes associated with wound healing occurs.(6) A general guideline is that scar revision is considered appropriate after 6 to 12 months, when type I collagen has largely replaced type III collagen and the general extent of scar formation is apparent.(7) Earlier scar revision may be considered in unfavorable scars in order to positively influence aesthetic and functional outcomes while also alleviating the patient's psychological distress. Unfavorable scars typically cross cosmetic subunits, do not fall within relaxed skin tension lines, and have more conspicuously disfiguring appearance. In contrast, scars that do not disrupt cosmetic subunits and that have favorable orientation relative to relaxed skin tension lines may have a satisfactory appearance at 1 year without any surgery, despite initial erythema and discoloration.

Patients who opt to pursue scar revision often have persistent psychological trauma associated with the original traumatic event, even if a significant period of time has elapsed between the original injury and the time of surgical consultation. The surgeon must therefore be attentive to the patient's concerns and ensure that the patient has realistic goals. The surgeon should impress upon the patient that complete elimination of scars is seldom, if ever, achieved, although improvement is often possible.(8) It is also important to stress the role for planned secondary procedures as part of the treatment. For example, scar revision with excision is often followed by steroid injection or contour correction with dermabrasion. Similarly, scar revision by serial excision involves sequential procedures.

In cases of significant psychological trauma, a specialist with relevant expertise may be consulted. When domestic violence has occurred, camouflaging may be particularly helpful as an interim strategy prior to definitive surgical therapy. Inquiring about the patient's social support system may afford the surgeon insights regarding potential factors that might adversely affect postoperative care. The patient should also understand the significant period of time required for healing.

\section{Surgical treatment}

A wide range of techniques are available for scar revision. Among these approaches are simple or serial excision, either with or without tissue expansion; irregularization through zplasty, w-plasty, m-plasty, or broken line closure; resurfacing with dermabrasion and lasers; minimally invasive approaches such as fillers and paralytic agents; and adjunctive techniques involving steroids, silicone sheeting, and cosmetics.(9) Each of these approaches is discussed in detail in the following section.

\subsection{General principles}

Atraumatic tissue handling, always important in surgery, assumes critical importance in revision surgery, where the wound edges are likely to have baseline vascular compromise. Toothed tissue forceps should be used, and tissue handling should be minimized. Use of skin hooks may diminish the need for traumatic tissue manipulation. Damp sponges may be used to help hydrate the skin edges, an approach that is of special value when using more 
labor intensive approaches, such as geometric broken line closures. Subdermal undermining lateral to wound margins is essential to achieving a tension free closure. Skin flap undermining is performed sharply, while elevating the flap atraumatically with skin hooks or toothed forceps. Layered closure is performed with tension placed upon the deep sutures to facilitate hypereversion.(10)

Adequate hemostasis is a prerequisite for successful scar revision. Collections of blood under a flap will predispose to infection and more visible scar. A bipolar cautery is preferred to monopolar cautery due to decreased thermal injury. Meticulous subcutaneous closure minimizes dead space and ensures a stable foundation for the overlying skin surface. Beveling of the skin incision can be used to improve wound margin eversion. For deep tissue and deep dermal closure on the face, 5-0 and 6-0 absorbable sutures are preferred (PDS or vicryl). For the skin, non-absorbable sutures (6-0 or 7-0 prolene or ethilon) are best due to their low tissue bioreactivity; however, these may be cumbersome to remove in hair-bearing areas. Absorbable suture, such as fast absorbing gut, is an acceptable alternative. Of note, the needle on ethilon suture can be too rough for use on delicate tissues such as eyelids or facial tissue in infants

\subsection{Surgical methods of irregularization}

A variety of methods are available for irregularization of scars, so as to camouflage scars. The eye is naturally attracted to straight lines, as such lines seldom appear in nature. Therefore, introducing irregularity affords significant benefit in making scars less conspicuous.

\subsubsection{Z-plasty}

The classic Z-plasty involves the transposition of two adjacent undermined triangle flaps, usually with angles of the $\mathrm{Z}$ measuring approximately 60 degrees. Classic Z-plasty and its variations are used for a variety of purposes, including to:

- Reorient a scar to lie parallel to RSTLs

- Increase scar length to lengthen a site of contracture

- Reorient a scar to lie in a more favorable position relative to cosmetic subunits

- Irregularize a scar by breaking a single line into segments

- Orient a skin incision away from an underlying scar to avoid a depressed scar

The simple Z-plasty is composed of 3 limbs, as shown in Figure 2. After transposition of the two triangle flaps, the middle limb is reoriented approximately 90 degrees if the Zplasty is 60 degrees. The extent of rotation and the lengthening both diminish with tighter Z-plasty configurations, as shown in Figure 3. The lengthening in one axis corresponds to shortening in the other axis with associated tissue distortion. As shown, a 30 degree Zplasty results in a $25 \%$ increase in length; a 45 degree Z-plasty results in a $50 \%$ increase in length; and a 60 degree Z-plasty results in a 75\% increase in length. A Z-plasty with a higher angle tends to create a standing cone deformity, whereas a Z-plasty with $<30$ degree angles has more risk of necrosis of the tips. The use of serial Z-plasty or compound Z-plasty can achieve effective scar lengthening with less tissue distortion and improved camouflage. The compound Z-plasty minimizes the number of incisions required for scar revision. Because a given scar can be reoriented in either of 2 directions, the surgeon must 
choose both the angle and the orientation for the Z-plasty that will most effectively align the scar with the RSTLs.
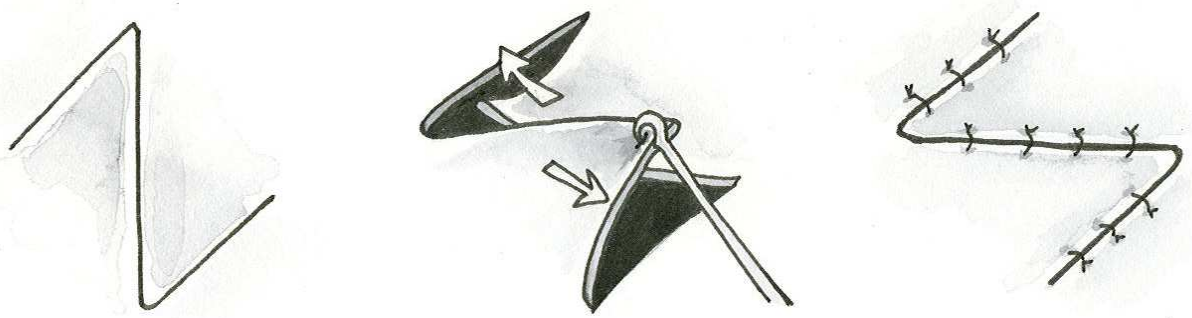

Fig. 2. Depiction of Z-plasty.

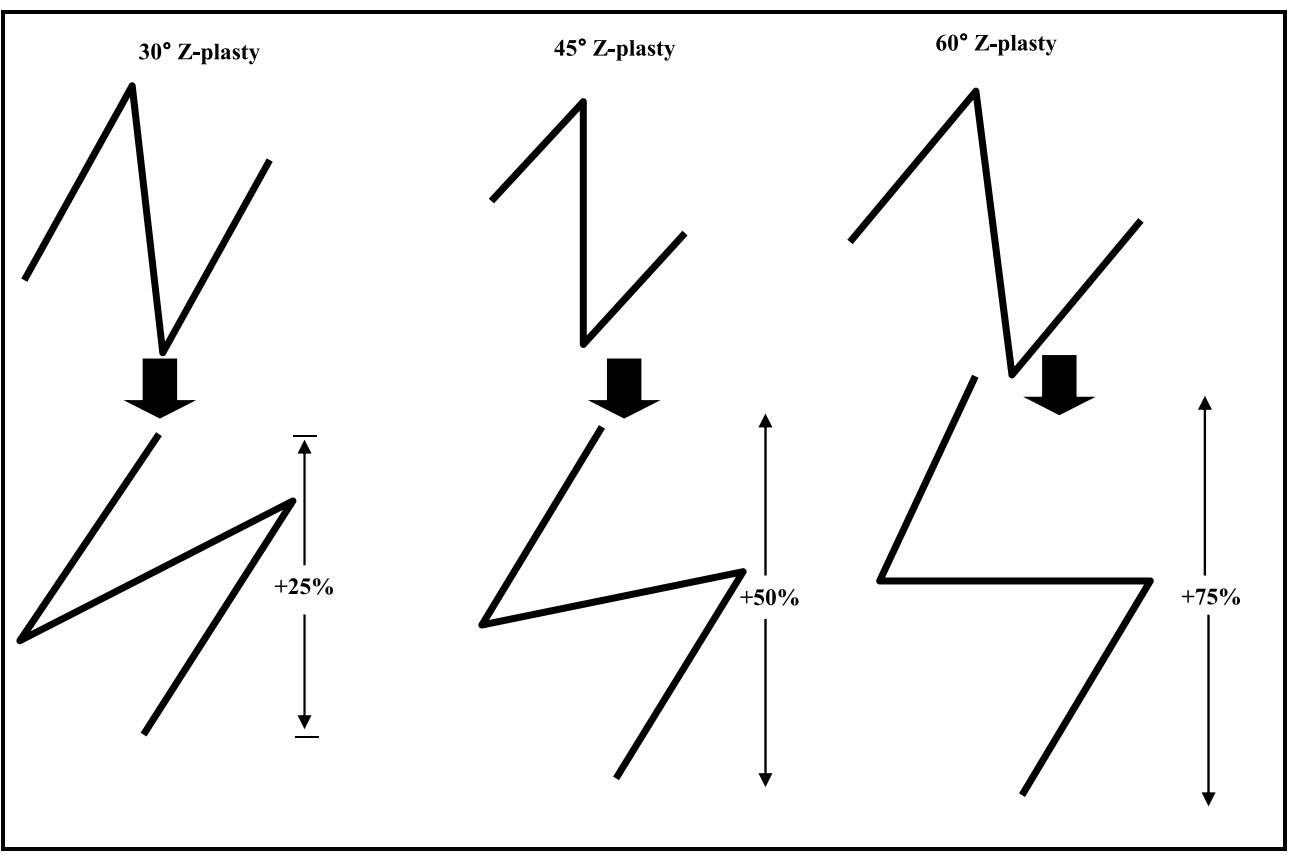

Fig. 3. With wider angle Z-plasty configurations, rotation and the lengthening both increase

The double-opposing Z-plasty, unequal triangle Z-plasty, and planimetric Z-plasty are other variants of the basic Z-plasty. The double opposing Z-plasty involves use of overlying Z- 
plasties in reverse orientation. This method can be used to redistribute volume and avoid placing multiple layers of closure over a single line of tension. This approach has been most widely applied in cleft palate surgery, although it has also found application for treatment of cervical webs, using the platysma for the deep Z-plasty and the skin and subcutaneous fat for the superficial opposing Z-plasty. The unequal triangle Z-plasty involves a Z with nonparallel limbs and is useful when it is desirable to transpose unequal tissue areas. Planimetric Z-plasty entails excising the excess elevated tissue (dog ears) that is produced with standard Z-plasty on a flat surface. It is used for scar irregularization and limited skin elongation on planar surfaces.

\subsubsection{W-plasty}

The W-plasty (Figure 4) is most useful in changing a linear scar into an irregular scar .(11) It can also be useful in converting a curvilinear scar into an irregular scar that might otherwise be predisposed to a trap-door type deformity. The length of the limbs in W-plasty should be less than $6 \mathrm{~mm}$. When using a curved W-plasty, the triangles of the outer limb must be wider than those of the inner limb in order that the tissue edges interlock properly. One advantage of the W-plasty is that it avoids the scar lengthening seen with Z-plasty. In addition, this W-plasty may be more amenable to orientation of the scar within RSTLs. However, the repetitive zig-zag pattern of $\mathrm{W}$-plasty is often more readily discernible to the eye than more irregular Z-plasty. Figure 5 compares a curved serial Z-plasty against a curved W-plasty.
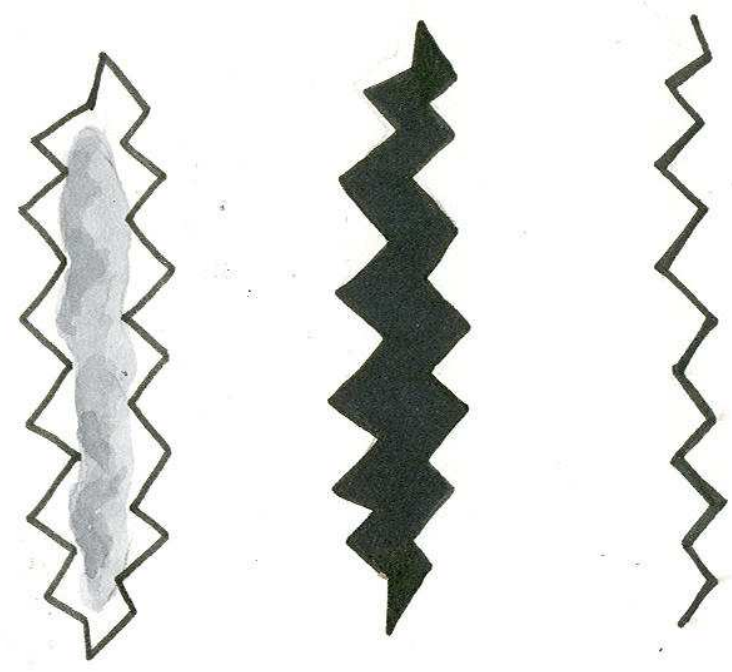

Fig. 4. W-plasty 

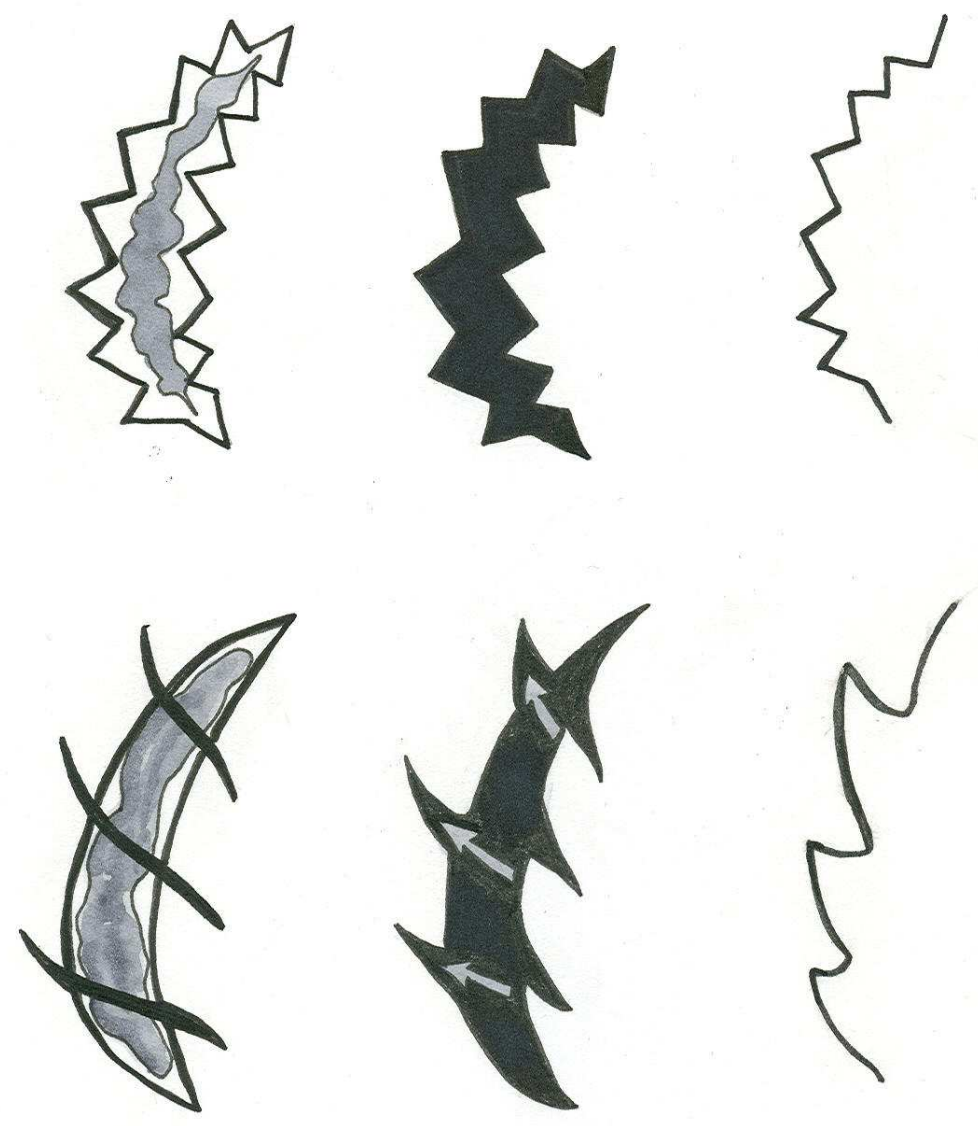

Fig. 5. Comparison of W-plasty versus serial Z-plasty

\subsubsection{Geometric broken line closure}

The geometric broken line closure is a variant of the W-plasty, in which a linear scar is rendered irregular by use of a mixture of triangles, squares, rectangles, and/or circles (Figure 6).(12) The geometric broken line closure is more labor intensive to construct than the $\mathrm{W}$-plasty given its varied design but yields a less visually perceptible result. The geometric shapes are intended to have a random sequence that interlocks on upper and lower sides. When the rectangles or squares within the geometric broken line closure are perpendicular to RSTLs, use of extra triangles may minimize any unfavorable appearance. 

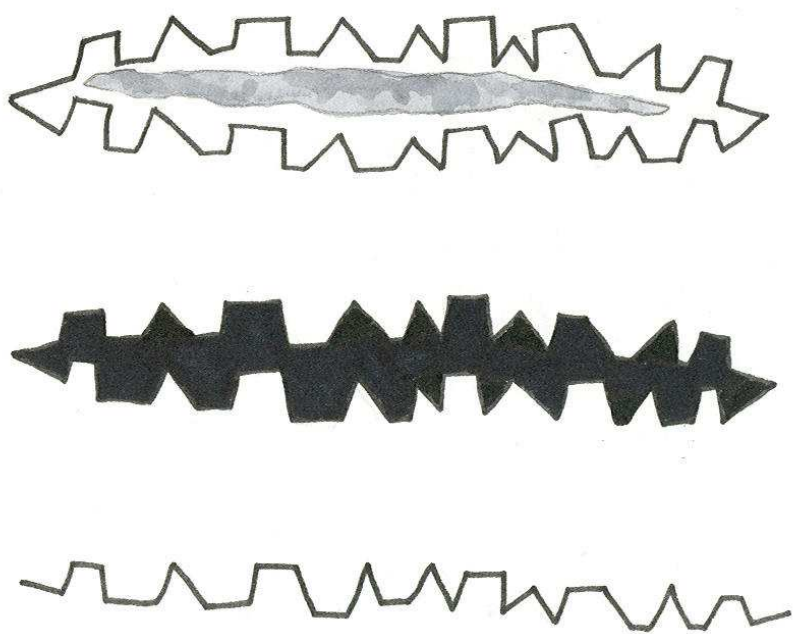

Fig. 6. Geometric Broken Line Closure

\subsubsection{M-plasty}

The M-plasty (Figure 7) minimizes the loss of surrounding healthy tissue at the site of a scar and also can minimize the length of the scar. When compared to the simple ellipse excision, the loss of healthy, normal tissue is decreased by approximately $50 \%$. The price paid for this preservation of healthy tissue is having two limbs at each pole of the M-plasty. The M-plasty is constructed by diminishing the distance from the midpoint of the wound to the lateral extents of the excision. By advancing the lateral triangles of tissue into the wound in a V-Y advancement fashion, the scar is shortened.
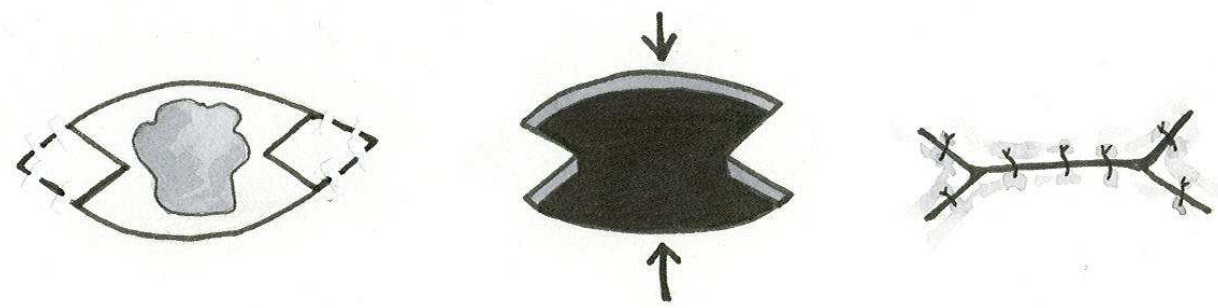

Fig. 7. M-plasty

\subsection{Other surgical methods of scar revision}

A variety of other surgical approaches are also useful in scar revision. Serial excision of scar is a logical extension of simple excision of ellipses in RSTLs. In this approach, a wound that would not readily close following complete excision is excised in multiple separate sittings to avoid undue stretch on the skin. Tissue expansion followed by excision may circumvent the need for serial excision if a sufficient area of skin for closure is created by the expander. 
Usually 2 expanders are required to achieve the desired degree of skin expansion. The major risks of tissue expanders are infection and unintended trauma to the skin from distention.(13) A V to Y advancement flap (Figure 8) allows for recruitment of excess tissue from laterally and proximally into an area that has been shortened by contracture. This method is also useful when a soft tissue defect needs to be reconstructed. It is sometimes preferable to excise an entire cosmetic subunit before proceeding with reconstruction using a local flap.(14)
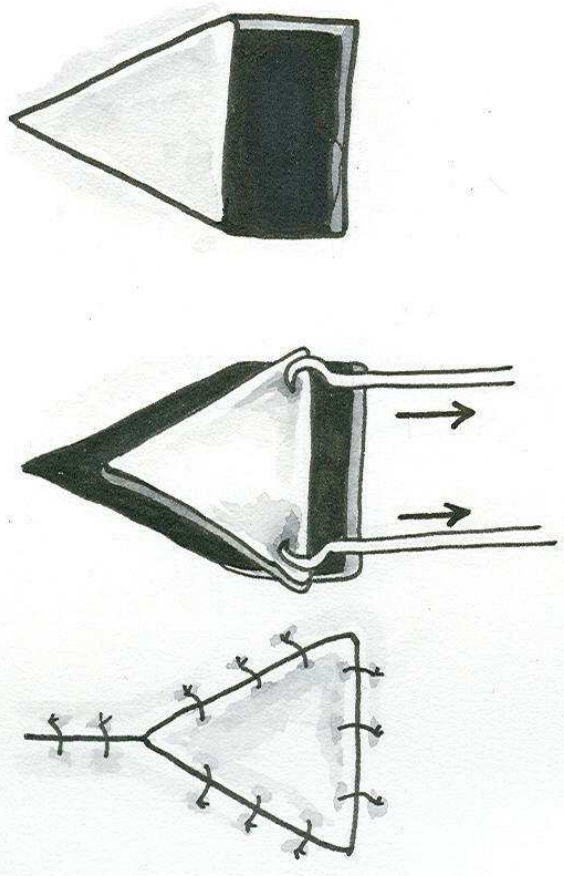

Fig. 8. V to $\mathrm{Y}$ advancement

\subsection{Special considerations related to subsite}

Each area of the head and neck has distinctive features with corresponding implications for the approach to scar revision. The various facial subsites differ in terms of RSTL orientation, solar exposure, skin thickness, pilosebaceous density, and muscle movement. The forehead, eyebrows, cheeks, nasolabial fold, and mentum are discussed below because of the special considerations that come into play for these areas.

\subsubsection{Forehead}

While simple fusiform excision yield favorable results in the upper forehead, at the junction of the forehead and glabella the RSTLs are virtually perpendicular. This orientation corresponds to the perpendicular orientation of corrugator and frontalis fibers. Scar revision in this area may require a combination a Z-plasty to reorient scars and irregularization with $\mathrm{W}$-plasty. Botulinum toxin may attenuate the wrinkles of this area. 


\subsubsection{Eyebrow}

The eyebrow region is a frequent area of unfavorable scarring that also warrants special consideration. Due to the prominence of the supraorbital rim, this site is prone to trauma in continuity with the forehead. Blunt trauma to this area may results in the underlying bone cutting the skin from beneath, as when the impact of a boxer's glove causes skin to shear against the underlying bone. This extensive soft tissue trauma predisposes to a significantly widened scar. While vertical incisions are commonly used elsewhere in scar revision, a beveled incision is needed in the eyebrow. The shafts of hair follicles are oriented obliquely; therefore, incisions made perpendicular to the skin are more likely to result in alopecia than beveled incisions that run parallel to the hair follicles. W-plasty is useful in camouflaging long, linear scars. Care must be taken to align the hairs when the eyebrow is divided by a scar.

\subsubsection{Cheek, nasolabial fold, and mentum}

The cheek, nasolabial fold, and mentum are also important areas in scar revision. The RSTLs of the cheek run from the zygoma to the mandible in a curved fashion. Many scars in this area run opposite the RSTLs and therefore require the use of a serial Z-plasty approach. When scars run parallel to the direction of RSTLs, a W-plasty will achieve excellent cosmesis. A terminal Z-plasty may achieve further irregularization.

The nasolabial fold is extremely useful in scar camouflage, and Z-plasty can be used to excellent advantage to reorient scars along the RSTLs. Only one of the two possible combinations of Z-plasty will yield an optimal cosmetic result, with the lateral limbs nearest the direction of the RSTLs. Scars along the mentum are effectively managed with W-plasty or Z-plasty for scars running parallel and oblique to RSTLs, respectively.

\subsection{Adjunctive treatments}

A variety of adjunctive techniques are available to assist in late wound management and scar camouflage. Many of these approaches are most effective when used as part of a surgical regimen, although some may prove useful alone. An important aspect of scar minimization is optimal postoperative care. This includes wound compression immediately following the procedure (such as using silicone sheets or micropore tape), UV protection (especially important in the first year after the procedure), and smoking cessation.

\subsubsection{Dermabrasion and lasers}

Dermabrasion and Laser skin resurfacing can be used to correct skin contour irregularities. Dermabrasion is useful to level a scar, to modify the texture of a scar, or to improve camouflage through blending with surrounding skin. It is typically performed approximately 6 to 8 weeks after W-plasty, Z-plasty, or geometric broken line closure.(15) Preoperative treatment with Retin-A will alleviate scarring, and antiviral therapy is indicated for patients with a history of herpetic infection. Care must be taken to avoid deep penetration into the reticular dermis, as excessive depth of dermabrasion is associated with risk of melanocyte loss and resulting permanent hypopigmentation. The adverse effects of dermabrasion on pigmentation are less significant in individuals with fairer skin. An occlusive dressing and moist ointment with regular cleansing will facilitate reepithelization. Hyperpigmented areas can be addressed with depigmentation agents, including hydroquinone, which blocks the production of melanin. This is available in $2 \%$ 
concentrations over the counter or $4 \%$ concentrations by prescription, with stronger concentrations being more effective but more prone to local skin irritation. Azelaic acid and kojic acid are two other effective depigmentation agents. Depressed scars may be ameliorated with use of fat/dermis grafts or allograft dermal matrix grafts. CO2 and Erbium lasers are also used for resurfacing. Lasers induce collagen reorganization and can thereby enhance camouflage, although variable depth of thermal damage and the potential for hypopigmentation are risks.(16) Intense pulsed light, KTP laser, and ND:YAG are among the techniques that have been used for vascular and pigmentary irregularities. $(17 ; 18)$

\subsubsection{Minimally invasive treatments}

Noninvasive resurfacing can be achieved using fillers. Improved symmetry can be achieved with administration of botulinum toxin to weaken one side of the face if the contralateral side is weak or paralyzed. Plucking of brows may also camouflage irregularities. Cosmetics, hairstyling, and hair replacement have all been used to enhance results. Makeup, tattoos, and prosthetics also can find useful application. Aestheticians are particularly helpful in the postoperative period, both to improve appearance during healing and to prevent erythema from sun exposure. Aestheticians also may treat irregularities that are not amenable to surgical revision.

\section{Special considerations: Hypertrophic and keloid scars}

Hypertrophic scars and keloids are generally accepted to occur more commonly in young, darkly pigmented individuals. Clinically, hypertrophic scars exhibit excessive deposition within the scar, whereas keloid scars overgrow the original margins of an incision to involve adjacent tissues. Ultrastructurally, hypertrophic scars have parallel alignment of collagen sheets, whereas keloids have disorganized sheets. Keloids also demonstrate a greater increase in collagenase and proline hydroxylase than do hypetrophic scars.

Although a variety of nonsurgical treatments have been investigated for management of hypertophic scars and keloids, the most common approach is primary excision with serial injection of steroid. Other approaches have included serial excision, carbon dioxide laser excision, and use of skin grafting. Topical application of silicone sheeting over sites of keloid formation has been shown to be beneficial in some series, although the mechanism by which sheeting may improve scar outcomes remains uncertain.(19) Benefit may be related to the improved hydration of tissues associated with this approach, as nonsilicone gel dressings may have similar efficacy.(20) Pulsed-dye lasers have been found effective for hypertrophic scars.(21) Other methods, including creams/vitamins, pressure dressings, and interferons have also been suggested. Thus there are a variety of options for treatment of scars. The role of compression garments, silicone sheets, scar massage and ultrasound should also be considered.

\section{Concluding remarks}

Late wound management after trauma includes surgical camouflaging of the aesthetically unacceptable scar and correction of functional impairments related to aberrant wound healing. The surgeon must remember that patients undergoing these procedures may harbor a significant emotional component to their injury. Optimal results are achieved through an in depth understanding of the mechanisms of scar formation and application of the optimal surgical technique, taking into consideration the characteristics of the site. 


\section{References}

[1] Kaplan B, Potter T, Moy RL, Kaplan B, Potter T, Moy RL. Scar revision. Dermatologic Surgery 1997; 23(6):435-442.

[2] Zakkak TB, Griffin JE, Jr., Max DP, Zakkak TB, Griffin JEJ, Max DP. Posttraumatic scar revision: a review and case presentation. Journal of Cranio-Maxillofacial Trauma 1998; 4(1):35-41.

[3] Thomas JR, Ehlert TK, Thomas JR, Ehlert TK. Scar revision and camouflage. Otolaryngologic Clinics of North America 1990; 23(5):963-973.

[4] Kokoska MS, Thomas JR. Scar Revision. In: Papel ID, editor. Facial Plastic and Reconstructive Surgery. New York: Thieme, 2002: 55-60.

[5] Schweinfurth JM, Fedok F, Schweinfurth JM, Fedok F. Avoiding pitfalls and unfavorable outcomes in scar revision. Facial Plastic Surgery 2001; 17(4):273-278.

[6] Goslen JB, Goslen JB. Wound healing for the dermatologic surgeon. Journal of Dermatologic Surgery \& Oncology 1988; 14(9):959-972.

[7] Thomas JR, Prendiville S, Thomas JR, Prendiville S. Update in scar revision. Facial Plastic Surgery Clinics of North America 2002; 10(1):103-111.

[8] Moran ML, Moran ML. Scar revision. Otolaryngologic Clinics of North America 2001; 34(4):767-780.

[9] Thomas JR, Mobley SR. Scar Revision and Camouflage. In: Cummings CW, Flint PW, Harker LA, Haughey BH, Richardson MA, Robbins KT et al., editors. Cummings Otolaryngology Head \& Neck Surgery. Philadelphia: Elsevier, 2005: 572-581.

[10] Zide MF, Zide MF. Scar revision with hypereversion. Journal of Oral \& Maxillofacial Surgery 1996; 54(9):1061-1067.

[11] Kelleher JC, Kelleher JC. W-plasty scar revision and its extended use. Clinics in Plastic Surgery 1977; 4(2):247-254.

[12] Harnick DB, Harnick DB. Broken geometrical pattern used for facial scar revision. Laryngoscope 1984; 94(6):841-842.

[13] Mostafapour SP, Murakami CS, Mostafapour SP, Murakami CS. Tissue expansion and serial excision in scar revision. Facial Plastic Surgery 2001; 17(4):245-252.

[14] Clark JM, Wang TD, Clark JM, Wang TD. Local flaps in scar revision. [Facial Plastic Surgery 2001; 17(4):295-308.

[15] Harmon CB, Zelickson BD, Roenigk RK, Wayner EA, Hoffstrom B, Pittelkow MR et al. Dermabrasive scar revision. Immunohistochemical and ultrastructural evaluation. Dermatologic Surgery 1995; 21(6):503-508.

[16] Alster T, Zaulyanov-Scanlon L, Alster T, Zaulyanov-Scanlon L. Laser scar revision: a review. [Review] [46 refs]. Dermatologic Surgery 2007; 33(2):131-140.

[17] Cassuto D, Emanuelli G, Cassuto D, Emanuelli G. Non-ablative scar revision using a long pulsed frequency doubled Nd:YAG laser. Journal of Cosmetic \& Laser Therapy 2003; 5(3-4):135-139.

[18] Westine JG, Lopez MA, Thomas JR, Westine JG, Lopez MA, Thomas JR. Scar revision. Facial Plastic Surgery Clinics of North America 2005; 13(2):325-331.

[19] Katz BE, Katz BE. Silicone gel sheeting in scar therapy. Cutis 1995; 56(1):65-67.

[20] de Oliveira GV, Nunes TA, Magna LA, Cintra ML, Kitten GT, Zarpellon S et al. Silicone versus nonsilicone gel dressings: a controlled trial. Dermatologic Surgery 2001; 27(8):721-726.

[21] Bradley DT, Park SS, Bradley DT, Park SS. Scar revision via resurfacing. Facial Plastic Surgery 2001; 17(4):253-262. 


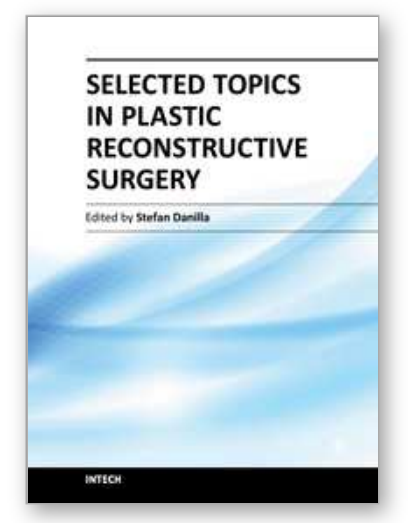

\author{
Selected Topics in Plastic Reconstructive Surgery \\ Edited by Dr Stefan Danilla
}

ISBN 978-953-307-836-6

Hard cover, 242 pages

Publisher InTech

Published online 20, January, 2012

Published in print edition January, 2012

Plastic Surgery is a fast evolving surgical specialty. Although best known for cosmetic procedures, plastic surgery also involves reconstructive and aesthetic procedures, which very often overlap, aiming to restore functionality and normal appearance of organs damaged due to trauma, neoplasm, ageing tissue or iatrogenesis. First reconstructive procedures were described more than 3000 years ago by Indian surgeons that reconstructed nasal deformities caused by nose amputation as a form of punishment. Nowadays, many ancient procedures are still used like the Indian forehead flap for nasal reconstruction, but as with all fields of medicine, the advances in technology and research have dramatically affected reconstructive surgery.

\title{
How to reference
}

In order to correctly reference this scholarly work, feel free to copy and paste the following:

Michael J. Brenner and Jennifer L. Nelson (2012). Scar Revision and Secondary Reconstruction for Skin Cancer, Selected Topics in Plastic Reconstructive Surgery, Dr Stefan Danilla (Ed.), ISBN: 978-953-307-836-6, InTech, Available from: http://www.intechopen.com/books/selected-topics-in-plastic-reconstructivesurgery/scar-revision-and-secondary-reconstruction-for-skin-cancer

\section{INTECH}

open science | open minds

\section{InTech Europe}

University Campus STeP Ri Slavka Krautzeka 83/A 51000 Rijeka, Croatia Phone: +385 (51) 770447

Fax: +385 (51) 686166 www.intechopen.com

\section{InTech China}

Unit 405, Office Block, Hotel Equatorial Shanghai No.65, Yan An Road (West), Shanghai, 200040, China 中国上海市延安西路65号上海国际贵都大饭店办公楼 405 单元 Phone: +86-21-62489820

Fax: $+86-21-62489821$ 
(C) 2012 The Author(s). Licensee IntechOpen. This is an open access article distributed under the terms of the Creative Commons Attribution 3.0 License, which permits unrestricted use, distribution, and reproduction in any medium, provided the original work is properly cited. 